\title{
A FIXED POINT APPROACH TO THE STABILITY OF THE QUADRATIC-ADDITIVE FUNCTIONAL EQUATION
}

\author{
SUN SOOK JIN ${ }^{a}$ AND YANG-Hi LEE ${ }^{b}$
}

Abstract. We investigate the stability of the functional equation

$$
\begin{aligned}
& f(x+y+z+w)+2 f(x)+2 f(y)+2 f(z)+2 f(w)-f(x+y) \\
& -f(x+z)-f(x+w)-f(y+z)-f(y+w)-f(z+w)=0
\end{aligned}
$$

by using a fixed point theorem in the sense of L. Cădariu and V. Radu.

\section{INTRODUCTION}

In 1940, S. M. Ulam [18] raised a question concerning the stability of homomorphisms:

"Given a group $G_{1}$, a metric group $G_{2}$ with the metric $d(\cdot, \cdot)$, and a positive number $\varepsilon$, does there exist a $\delta>0$ such that if a mapping $f: G_{1} \rightarrow G_{2}$ satisfies the inequality $d(f(x y), f(x) f(y))<\delta$ for all $x, y \in G_{1}$ then there exists a homomorphism $F: G_{1} \rightarrow G_{2}$ with $d(f(x), F(x))<\varepsilon$ for all $x \in G_{1}$ ?"

When this problem has a solution, we say that the homomorphisms from $G_{1}$ to $G_{2}$ are stable. In the next year, D. H. Hyers [7] gave a partial solution of Ulam's problem for the case of approximate additive mappings under the assumption that $G_{1}$ and $G_{2}$ are Banach spaces. Hyers' result was generalized by T. Aoki [1] for additive mappings and by Th. M. Rassias [16] for linear mappings by considering the stability problem with unbounded Cauchy differences. The paper of Th. M. Rassias had much influence in the development of stability problems. The terminology Hyers-UlamRassias stability originated from this historical background. During the last decades, the stability problems of functional equations have been extensively investigated by a number of mathematicians, see [6], [8]-[14].

Received by the editors June 16, 2011. Revised October 28, 2011. Accepted November 8, 2011. 2000 Mathematics Subject Classification. Primary 39B52.

Key words and phrases. Hyers-Ulam-Rassias stability, fixed point method, quadratic-additive functional equation. 
Almost all subsequent proofs, in this very active area, have used Hyers' method of [7]. Namely, the mapping $F$, which is the solution of a functional equation, is explicitly constructed, starting from the given mapping $f$, by the formulae $F(x)=\lim _{n \rightarrow \infty} \frac{1}{2^{n}} f\left(2^{n} x\right)$ or $F(x)=\lim _{n \rightarrow \infty} 2^{n} f\left(\frac{x}{2^{n}}\right)$. We call it a direct method. In 2003, L. Cădariu and V. Radu [2] observed that the existence of the solution $F$ for a functional equation and the estimation of the difference with the given mapping $f$ can be obtained from the fixed point theory alternative. This method is called $a$ fixed point method. In 2004, they [4] applied this method to prove stability theorems of the Cauchy functional equation

$$
f(x+y)-f(x)-f(y)=0 .
$$

In 2003, they [3] obtained the stability of the quadratic functional equation

$$
f(x+y)+f(x-y)-2 f(x)-2 f(y)=0
$$

by using the fixed point method. Notice that if we consider $f_{1}, f_{2}: \mathbb{R} \rightarrow \mathbb{R}$ defined by $f_{1}(x)=a x$ and $f_{2}(x)=a x^{2}$, where $a$ is a real constant, then $f_{1}$ satisfies the equation (1.1) and $f_{2}$ holds (1.2), respectively. We say a solution of (1.1) an additive map and a mapping satisfying (1.2) is called a quadratic map. Now we consider the following functional equation:

$$
\begin{aligned}
& f(x+y+z+w)+2 f(x)+2 f(y)+2 f(z)+2 f(w)-f(x+y) \\
& -f(x+z)-f(x+w)-f(y+z)-f(y+w)-f(z+w)=0
\end{aligned}
$$

which is called the quadratic-additive functional equation. The function $f: \mathbb{R} \rightarrow \mathbb{R}$ defined by $f(x)=a x^{2}+b x$ satisfies this functional equation, where $a, b$ are real constants. We call a solution of (1.3) a quadratic-additive mapping. In 2004, Chang et al [5] obtained a stability of the functional equation (1.3) by handling the odd part and the even part of the given mapping $f$, respectively. In their processing, they needed to take an additive map $A$ which is close to the odd part $\frac{f(x)-f(-x)}{2}$ of $f$ and a quadratic map $Q$ which is approximate to the even part $\frac{f(x)+f(-x)}{2}$ of it, and then combining $A$ and $Q$ to prove the existence of a quadratic-additive mapping $F$ which is close to the given mapping $f$.

In this paper, we will prove the stability of the quadratic-additive functional equation (1.3) by using a fixed point theorem. In the previous results of stability problems of (1.3), as we mentioned above, they had to get a solution by using the direct method to the odd part and the even part, respectively. Instead of splitting the given mapping $f: X \rightarrow Y$ into two parts, in this paper, we can take the desired 
solution $F$ at once. Precisely, we introduce a strictly contractive mapping with Liptshitz constant $0<L<1$. Using a fixed point theorem in the sense of L. Cădariu and V. Radu, together with suitable conditions, we can show that the contractive mapping has the fixed point. Actually the fixed point $F$ becomes the precise solution of (1.3). In section 2, we prove several stability results of the functional equation (1.3) using a fixed point theorem, see Theorem 2.3 and Theorem 2.5. In section 3, we use the results in the previous sections to get a stability of the Cauchy functional equation (1.1) and that of the quadratic functional equation (1.2), respectively.

\section{Main Results}

We recall the following result of the fixed point theorem by Margolis and Diaz.

Theorem 2.1 ([15, 17]). Suppose that a complete generalized metric space $(X, d)$, which means that the metric d may assume infinite values, and a strictly contractive mapping $J: X \rightarrow X$ with the Lipschitz constant $0<L<1$ are given. Then, for each given element $x \in X$, either

$$
d\left(J^{n} x, J^{n+1} x\right)=+\infty, \forall n \in \mathbb{N} \cup\{0\},
$$

or there exists a nonnegative integer $k$ such that:

(1) $d\left(J^{n} x, J^{n+1} x\right)<+\infty$ for all $n \geq k$;

(2) the sequence $\left\{J^{n} x\right\}$ is convergent to a fixed point $y^{*}$ of $J$;

(3) $y^{*}$ is the unique fixed point of $J$ in $Y:=\left\{y \in X, d\left(J^{k} x, y\right)<+\infty\right\}$;

(4) $d\left(y, y^{*}\right) \leq(1 /(1-L)) d(y$, Jy) for all $y \in Y$.

Throughout this paper, let $\mathrm{V}$ be a (real or complex) linear space and $Y$ a Banach space. For a given mapping $f: V \rightarrow Y$, we use the following abbreviation

$$
\begin{aligned}
D f(x, y, z, w):= & f(x+y+z+w)+2 f(x)+2 f(y)+2 f(z)+2 f(w)-f(x+y) \\
& -f(x+z)-f(x+w)-f(y+z)-f(y+w)-f(z+w)
\end{aligned}
$$

for all $x, y, z, w \in V$. If $f$ is a solution of the functional equation $D f \equiv 0$, see (1.3), we call it a quadratic-additive mapping. We first prove the following lemma.

Lemma 2.2. If $f: V \rightarrow Y$ is a mapping such that $D f(x, y, z, w)=0$ for all $x, y, z, w \in V \backslash\{0\}$, then $f$ is a quadratic-additive mapping.

Proof. By (1.3), we enough to show that $D f \equiv 0$. By choosing $x \in V \backslash\{0\}$, we get

$$
f(0)=\frac{1}{3}(D f(x, x, x, x)+D f(-x,-x,-x,-x)
$$




$$
+D f(2 x, 2 x,-2 x,-2 x)-2 D f(x, x,-x,-x))=0
$$

and

$$
\begin{aligned}
D f(x, y, z, 0)= & D f(2 x, y, z,-x)-D f(2 x, y,-x,-x)+D f(2 x, y, x,-x) \\
& -D f(2 x, z,-x,-x)+D f(2 x, z, x,-x)-2 D f(2 x, x, x,-x) \\
& +D f(x,-x,-x,-x)=0
\end{aligned}
$$

for all $x, y, z \in V \backslash\{0\}$. Moreover, it is easy to prove that

$$
D f(x, y, 0,0)=D f(x, 0,0,0)=0
$$

for all $x, y \in V \backslash\{0\}$. By the symmetry of the variables $x, y, z, w$, this implies the desired result.

In the following theorem, we can prove the stability of the functional equation (1.3) using the fixed point theorem.

Theorem 2.3. Let $f: V \rightarrow Y$. Suppose that we have a function $\varphi:(V \backslash\{0\})^{4} \rightarrow$ $[0, \infty)$ such that

$$
\|D f(x, y, z, w)\| \leq \varphi(x, y, z, w)
$$

for all $x, y, z, w \in V \backslash\{0\}$, which has the property

$$
\varphi(2 x, 2 y, 2 z, 2 w) \leq 2 L \varphi(x, y, z, w)
$$

for all $x, y, z, w \in V \backslash\{0\}$ and for a fixed positive real number $0<L<1$. Then there exists a unique quadratic-additive mapping $F: V \rightarrow Y$ such that

$$
\|f(x)-f(0)-F(x)\| \leq \frac{3 \psi(x)}{16\left(1-\max \left\{L, \frac{1}{2}\right\}\right)}
$$

for all $x \in V \backslash\{0\}$, where $\psi: V \backslash\{0\} \rightarrow[0, \infty)$ is defined by

$$
\psi(x):=\varphi(x, x, x,-x)+\varphi(-x,-x,-x, x)+2\|f(0)\| .
$$

In particular, $F$ is represented by

$$
F(x)=\lim _{n \rightarrow \infty}\left(\frac{f\left(2^{n} x\right)+f\left(-2^{n} x\right)}{2 \cdot 4^{n}}+\frac{f\left(2^{n} x\right)-f\left(-2^{n} x\right)}{2^{n+1}}\right)
$$

for all $x \in V$. Moreover, if $0<L<\frac{1}{2}$ and $\varphi(x, y, z, w)$ is continuous, then $f$ is itself a quadratic-additive mapping. 
Proof. It follows from (2.2) that

$$
\lim _{n \rightarrow \infty} \frac{\varphi\left(2^{n} x, 2^{n} y, 2^{n} z, 2^{n} w\right)}{2^{n}}=0
$$

for all $x, y, z, w \in V \backslash\{0\}$. Let $S$ be the set of all mappings $g: V \rightarrow Y$ with $g(0)=0$. If we consider the mapping $\tilde{f}=f-f(0)$, then $\tilde{f} \in S$. We introduce a generalized metric on $S$ by

$$
d(g, h):=\inf \left\{K \in \mathbb{R}^{+} \mid\|g(x)-h(x)\| \leq K \psi(x) \text { for all } x \in V \backslash\{0\}\right\}
$$

where $\psi$ is defined as (2.4). Observe that $\psi(x)=\psi(-x)$ and $\frac{\psi(2 x)}{2} \leq \max \left\{L, \frac{1}{2}\right\} \psi(x)$ for all $x \in V \backslash\{0\}$. It is easy to show that $(S, d)$ is a generalized complete metric space. Now we consider the mapping $J: S \rightarrow S$, which is defined by

$$
J g(x):=\frac{g(2 x)-g(-2 x)}{4}+\frac{g(2 x)+g(-2 x)}{8}
$$

for all $x \in V$. Notice that

$$
J^{n} g(x)=\frac{g\left(2^{n} x\right)-g\left(-2^{n} x\right)}{2^{n+1}}+\frac{g\left(2^{n} x\right)+g\left(-2^{n} x\right)}{2 \cdot 4^{n}}
$$

for all $n \in \mathbb{N}$ and $x \in V$. Let $g, h \in S$ and let $K \in[0, \infty]$ be an arbitrary constant with $d(g, h) \leq K$. From the definition of $d$, we have

$$
\begin{aligned}
\|J g(x)-J h(x)\| & =\frac{3}{8} \|\left(g(2 x)-h(2 x)\left\|+\frac{1}{8}\right\|(g(-2 x)-h(-2 x) \|\right. \\
& \leq \frac{1}{2} K \psi(2 x) \leq \max \left\{L, 2^{-1}\right\} K \psi(x)
\end{aligned}
$$

for all $x \in V \backslash\{0\}$, which implies that

$$
d(J g, J h) \leq \max \left\{L, 2^{-1}\right\} d(g, h)
$$

for any $g, h \in S$. That is, $J$ is a strictly contractive self-mapping of $S$ with the Lipschitz constant $\max \left\{L, \frac{1}{2}\right\}$. Moreover, by (2.1), we see that

$$
\begin{aligned}
\|\tilde{f}(x)-J \tilde{f}(x)\| & =\frac{1}{16}\|3 D f(x, x, x,-x)-D f(-x,-x,-x, x)-6 f(0)\| \\
& \leq \frac{3}{16} \psi(x)
\end{aligned}
$$

for all $x \in V \backslash\{0\}$. It means that $d(\tilde{f}, J \tilde{f}) \leq \frac{3}{16}<\infty$ by the definition of $d$. Therefore, according to Theorem 2.1, the sequence $\left\{J^{n} \tilde{f}\right\}$ converges to the unique fixed point 
$F: V \rightarrow Y$ of $J$ in the set $T=\{g \in S \mid d(\tilde{f}, g)<\infty\}$, which is represented by

$$
\begin{aligned}
F(x) & :=\lim _{n \rightarrow \infty}\left(\frac{\tilde{f}\left(2^{n} x\right)+\tilde{f}\left(-2^{n} x\right)}{2 \cdot 4^{n}}+\frac{\tilde{f}\left(2^{n} x\right)-\tilde{f}\left(-2^{n} x\right)}{2^{n+1}}\right) \\
& =\lim _{n \rightarrow \infty}\left(\frac{f\left(2^{n} x\right)+f\left(-2^{n} x\right)}{2 \cdot 4^{n}}+\frac{f\left(2^{n} x\right)-f\left(-2^{n} x\right)}{2^{n+1}}\right)
\end{aligned}
$$

for all $x \in V$, since $\lim _{n \rightarrow \infty} \frac{f(0)}{2 \cdot 4^{n}}=0$. Moreover, we get

$$
d(\tilde{f}, F) \leq \frac{1}{1-\max \left\{L, \frac{1}{2}\right\}} d(\tilde{f}, J \tilde{f}) \leq \frac{3}{16\left(1-\max \left\{L, \frac{1}{2}\right\}\right)}
$$

which implies (2.3). By the definition of $F$, together with (2.1) and (2.2), we have

$$
\begin{aligned}
\| & D F(x, y, z, w) \| \\
= & \lim _{n \rightarrow \infty} \| \frac{D f\left(2^{n} x, 2^{n} y, 2^{n} z, 2^{n} w\right)-D f\left(-2^{n} x,-, 2^{n} y,-2^{n} z,-2^{n} w\right)}{2^{n+1}} \\
& +\frac{D f\left(2^{n} x, 2^{n} y, 2^{n} z, 2^{n} w\right)+D f\left(-2^{n} x,-2^{n} y,-2^{n} z,-2^{n} w\right)}{2 \cdot 4^{n}} \| \\
\leq & \lim _{n \rightarrow \infty} \frac{2^{n}+1}{2 \cdot 4^{n}}\left(\varphi\left(2^{n} x, 2^{n} y, 2^{n} z, 2^{n} w\right)+\varphi\left(-2^{n} x,-2^{n} y,-2^{n} z,-2^{n} w\right)\right) \\
\leq & \lim _{n \rightarrow \infty} \frac{4^{n}+2^{n}}{2 \cdot 4^{n}} L^{n}(\varphi(x, y, z, w)+\varphi(-x,-y,-z,-w)) \\
=0 &
\end{aligned}
$$

for all $x, y, z, w \in V \backslash\{0\}$. From Lemma 2.2, we have proved that

$$
D F(x, y, z, w)=0
$$

for all $x, y, z, w \in V$. In particular, if $f(0)=0$, then we have

$$
\|J g(x)-J h(x)\| \leq \frac{1}{2} K \psi(2 x) \leq L K \psi(x)
$$

for all $x \in V \backslash\{0\}$. From this, we have

$$
d(J g, J h) \leq L d(g, h)
$$

for any $g, h \in S$ and

$$
\|f(x)-F(x)\| \leq \frac{3 \psi(x)}{16(1-L)}
$$

for all $x \in V \backslash\{0\}$. Now let $0<L<\frac{1}{2}$ and $\varphi$ be continuous. Since

$$
\begin{aligned}
\|f(0)\|= & \frac{1}{3} \| D f\left(2^{n} x, 2^{n} x, 2^{n} x, 2^{n} x\right)+D f\left(2^{n+1} x, 2^{n+1} x,-2^{n+1} x,-2^{n+1} x\right) \\
& +D f\left(-2^{n} x,-2^{n} x,-2^{n} x,-2^{n} x\right)-2 D f\left(2^{n} x, 2^{n} x,-2^{n} x,-2^{n} x\right) \|
\end{aligned}
$$




$$
\begin{aligned}
\leq & \frac{1}{3}\left(\varphi\left(2^{n} x, 2^{n} x, 2^{n} x, 2^{n} x\right)+\varphi\left(2^{n+1} x, 2^{n+1} x,-2^{n+1} x,-2^{n+1} x\right)\right. \\
& \left.+\varphi\left(-2^{n} x,-2^{n} x,-2^{n} x,-2^{n} x\right)+2 \varphi\left(2^{n} x, 2^{n} x,-2^{n} x,-2^{n} x\right)\right) \\
\leq & \frac{(2 L)^{n}}{3}(\varphi(x, x, x, x)+\varphi(-x,-x,-x,-x) \\
& +\varphi(2 x, 2 x,-2 x,-2 x)+2 \varphi(x, x,-x,-x))
\end{aligned}
$$

for all $n \in \mathbb{N}$ and for any fixed $x \in V \backslash\{0\}$, the last term of the above inequality tends to 0 as $n \rightarrow \infty$. This implies that $f(0)=0$. And we get

$$
\begin{aligned}
& \lim _{n \rightarrow \infty} \varphi\left(\left(a_{1} \cdot 2^{n}+a_{2}\right) x,\left(b_{1} \cdot 2^{n}+b_{2}\right) y,\left(c_{1} \cdot 2^{n}+c_{2}\right) z,\left(d_{1} \cdot 2^{n}+d_{2}\right) w\right) \\
& \quad \leq \lim _{n \rightarrow \infty}(2 L)^{n} \varphi\left(\left(a_{1}+\frac{a_{2}}{2^{n}}\right) x,\left(b_{1}+\frac{b_{2}}{2^{n}}\right) y,\left(c_{1}+\frac{c_{2}}{2^{n}}\right) z,\left(d_{1}+\frac{d_{2}}{2^{n}}\right) w\right) \\
& \quad=0 \cdot \varphi\left(a_{1} x, b_{1} y, c_{1} z, d_{1} w\right)=0
\end{aligned}
$$

for all $x, y, z, w \in V \backslash\{0\}$ and for any fixed integers $a_{1}, a_{2}, b_{1}, b_{2}, c_{1}, c_{2}, d_{1}, d_{2}$ with $a_{1}, b_{1}, c_{1}, d_{1} \neq 0$. Therefore, we obtain

$$
\begin{aligned}
3\|F(x)-f(x)\| \leq & \lim _{n \rightarrow \infty}\left(\left\|(D f-D F)\left(\left(2^{n}+1\right) x,-2^{n} x,-2^{n} x,-2^{n} x\right)\right\|\right. \\
& +\left\|(F-f)\left(\left(-2^{n+1}+1\right) x\right)\right\|+3\left\|(f-F)\left(-2^{n+1} x\right)\right\| \\
& \left.+6\left\|(F-f)\left(-2^{n} x\right)\right\|+2\left\|(F-f)\left(\left(2^{n}+1\right) x\right)\right\|\right) \\
\leq & \lim _{n \rightarrow \infty}\left(\varphi\left(\left(2^{n}+1\right) x,-2^{n} x,-2^{n} x,-2^{n} x\right)\right. \\
& \left.+\frac{3\left(\psi\left(\left(1-2^{n+1}\right) x\right)+3 \psi\left(2^{n+1} x\right)+2 \psi\left(\left(2^{n}+1\right) x\right)+6 \psi\left(2^{n} x\right)\right)}{16(1-L)}\right) \\
= & 0
\end{aligned}
$$

for all $x \in V \backslash\{0\}$. Since $f(0)=0=F(0)$, we have shown that $f \equiv F$. This completes the proof of this theorem.

Remark 2.4. In Theorem 2.3, if $\varphi$ satisfies the additional conditions $\varphi(x, y, z, w)=$ $\varphi(-x,-y,-z,-w)$ and $\varphi(x, y, z, w) \leq L^{\prime} \varphi(2 x, 2 y, 2 z, 2 w)$ for all $x, y, z, w \in V \backslash\{0\}$ with $0<L^{\prime}<1$, then

$$
\begin{aligned}
\|f(0)\|= & \lim _{n \rightarrow \infty} \frac{1}{3} \| D f\left(\frac{x}{2^{n}}, \frac{x}{2^{n}}, \frac{x}{2^{n}}, \frac{x}{2^{n}}\right)+D f\left(-\frac{x}{2^{n}},-\frac{x}{2^{n}},-\frac{x}{2^{n}},-\frac{x}{2^{n}}\right) \\
& +D f\left(\frac{2 x}{2^{n}}, \frac{2 x}{2^{n}},-\frac{2 x}{2^{n}},-\frac{2 x}{2^{n}}\right)-2 D f\left(\frac{x}{2^{n}}, \frac{x}{2^{n}},-\frac{x}{2^{n}},-\frac{x}{2^{n}}\right) \| \\
\leq & \lim _{n \rightarrow \infty} \frac{1}{3}\left(\varphi\left(\frac{x}{2^{n}}, \frac{x}{2^{n}}, \frac{x}{2^{n}}, \frac{x}{2^{n}}\right)+\varphi\left(-\frac{x}{2^{n}},-\frac{x}{2^{n}},-\frac{x}{2^{n}},-\frac{x}{2^{n}}\right)\right.
\end{aligned}
$$




$$
\begin{aligned}
&\left.+\varphi\left(\frac{2 x}{2^{n}}, \frac{2 x}{2^{n}},-\frac{2 x}{2^{n}},-\frac{2 x}{2^{n}}\right)+2 \varphi\left(\frac{x}{2^{n}}, \frac{x}{2^{n}},-\frac{x}{2^{n}},-\frac{x}{2^{n}}\right)\right) \\
& \leq \lim _{n \rightarrow \infty} \frac{L^{\prime n}}{3}(\varphi(x, x, x, x)+\varphi(-x,-x,-x,-x) \\
&+\varphi(2 x, 2 x,-2 x,-2 x)+2 \varphi(x, x,-x,-x))=0
\end{aligned}
$$

for all $x \in V \backslash\{0\}$. Since $\varphi$ satisfies $\varphi(x, y, z, w)=\varphi(-x,-y,-z,-w)$ for all $x, y, z, w \in V \backslash\{0\}$ and $f(0)=0$, we get

$$
\begin{aligned}
\|f(x)-J f(x)\| & =\frac{1}{16}\|3 D f(x, x, x,-x)-D f(-x,-x,-x, x)\| \\
& \leq \frac{1}{8} \psi(x)
\end{aligned}
$$

for all $x \in V \backslash\{0\}$, where $\psi: V \backslash\{0\} \rightarrow[0, \infty)$ is defined as Theorem 2.3. It means that $d(f, J f) \leq \frac{1}{8}<\infty$ by the definition of $d$. Therefore the inequality (2.3) can be replaced by the inequality

$$
\|f(x)-F(x)\| \leq \frac{\varphi(x, x, x,-x)}{4\left(1-L^{\prime}\right)}
$$

for all $x \in V \backslash\{0\}$.

We continue our investigation with the next result.

Theorem 2.5. Let $\varphi:(V \backslash\{0\})^{4} \rightarrow[0, \infty)$. Suppose that $f: V \rightarrow Y$ satisfies the inequality $\|D f(x, y, z, w)\| \leq \varphi(x, y, z, w)$ for all $x, y, z, w \in V \backslash\{0\}$. If there exists $0<L<1$ such that $\varphi$ has the property

$$
L \varphi(2 x, 2 y, 2 z, 2 w) \geq 4 \varphi(x, y, z, w)
$$

for all $x, y, z, w \in V \backslash\{0\}$, then there exists a unique quadratic-additive mapping $F: V \rightarrow Y$ such that

$$
\|f(x)-F(x)\| \leq \frac{L}{8(1-L)}(\varphi(x, x, x,-x)+\varphi(-x,-x,-x, x))
$$

for all $x \in V \backslash\{0\}$. In particular, $F$ is represented by

$$
F(x)=\lim _{n \rightarrow \infty}\left(2^{n-1}\left(f\left(\frac{x}{2^{n}}\right)-f\left(-\frac{x}{2^{n}}\right)\right)+\frac{4^{n}}{2}\left(f\left(\frac{x}{2^{n}}\right)+f\left(-\frac{x}{2^{n}}\right)\right)\right)
$$

for all $x \in V$.

Proof. Since for all $n \in \mathbb{N}$ and a fixed $x \in V \backslash\{0\}$

$$
\|f(0)\|=\frac{1}{3} \| D f\left(\frac{x}{2^{n}}, \frac{x}{2^{n}}, \frac{x}{2^{n}}, \frac{x}{2^{n}}\right)+D f\left(-\frac{x}{2^{n}},-\frac{x}{2^{n}},-\frac{x}{2^{n}},-\frac{x}{2^{n}}\right)
$$




$$
\begin{aligned}
& +\operatorname{Df}\left(\frac{2 x}{2^{n}}, \frac{2 x}{2^{n}},-\frac{2 x}{2^{n}},-\frac{2 x}{2^{n}}\right)-2 D f\left(\frac{x}{2^{n}}, \frac{x}{2^{n}},-\frac{x}{2^{n}},-\frac{x}{2^{n}}\right) \| \\
\leq & \frac{1}{3}\left(\varphi\left(\frac{x}{2^{n}}, \frac{x}{2^{n}}, \frac{x}{2^{n}}, \frac{x}{2^{n}}\right)+\varphi\left(-\frac{x}{2^{n}},-\frac{x}{2^{n}},-\frac{x}{2^{n}},-\frac{x}{2^{n}}\right)\right. \\
& \left.+\varphi\left(\frac{2 x}{2^{n}}, \frac{2 x}{2^{n}},-\frac{2 x}{2^{n}},-\frac{2 x}{2^{n}}\right)+2 \varphi\left(\frac{x}{2^{n}}, \frac{x}{2^{n}},-\frac{x}{2^{n}},-\frac{x}{2^{n}}\right)\right) \\
\leq & \frac{L^{n}}{3 \cdot 4^{n}}(\varphi(x, x, x, x)+\varphi(-x,-x,-x,-x) \\
& +\varphi(2 x, 2 x,-2 x,-2 x)+2 \varphi(x, x,-x,-x))
\end{aligned}
$$

letting $n \rightarrow \infty$ we have $f(0)=0$. Let the set $(S, d)$ be as in the proof of Theorem 2.3. Now we consider the mapping $J: S \rightarrow S$ defined by

$$
J g(x):=g\left(\frac{x}{2}\right)-g\left(-\frac{x}{2}\right)+2\left(g\left(\frac{x}{2}\right)+g\left(-\frac{x}{2}\right)\right)
$$

for all $g \in S$ and $x \in V$. Notice that

$$
J^{n} g(x)=2^{n-1}\left(g\left(\frac{x}{2^{n}}\right)-g\left(-\frac{x}{2^{n}}\right)\right)+\frac{4^{n}}{2}\left(g\left(\frac{x}{2^{n}}\right)+g\left(-\frac{x}{2^{n}}\right)\right)
$$

and $J^{0} g(x)=g(x)$ for all $x \in V$. Let $g, h \in S$ and let $K \in[0, \infty]$ be an arbitrary constant with $d(g, h) \leq K$. From the definition of $d$, we have

$$
\begin{aligned}
\|J g(x)-J h(x)\| & =3\left\|g\left(\frac{x}{2}\right)-h\left(\frac{x}{2}\right)\right\|+\left\|g\left(-\frac{x}{2}\right)-h\left(-\frac{x}{2}\right)\right\| \\
& \leq 4 K\left(\varphi\left(\frac{x}{2}, \frac{x}{2}, \frac{x}{2},-\frac{x}{2}\right)+\varphi\left(-\frac{x}{2},-\frac{x}{2},-\frac{x}{2}, \frac{x}{2}\right)\right) \\
& \leq \operatorname{LK}(\varphi(x, x, x,-x)+\varphi(-x,-x,-x, x))
\end{aligned}
$$

for all $x \in V$. So

$$
d(J g, J h) \leq L d(g, h)
$$

for any $g, h \in S$. That is, $J$ is a strictly contractive self-mapping of $S$ with the Lipschitz constant $L$. Also we see that

$$
\begin{aligned}
\|f(x)-J f(x)\| & =\frac{1}{2}\left\|-\operatorname{Df}\left(\frac{x}{2}, \frac{x}{2}, \frac{x}{2},-\frac{x}{2}\right)\right\| \\
& \leq \frac{1}{2}\left(\varphi\left(\frac{x}{2}, \frac{x}{2}, \frac{x}{2},-\frac{x}{2}\right)+\varphi\left(-\frac{x}{2},-\frac{x}{2},-\frac{x}{2}, \frac{x}{2}\right)\right) \\
& \leq \frac{L}{8}(\varphi(x, x, x,-x)+\varphi(-x,-x,-x, x))
\end{aligned}
$$

for all $x \in V \backslash\{0\}$, which implies that $d(f, J f) \leq \frac{L}{8}<\infty$. Therefore according to Theorem 2.1, the sequence $\left\{J^{n} f\right\}$ converges to the unique fixed point $F$ of $J$ in the 
set $T:=\{g \in S \mid d(f, g)<\infty\}$, which is represented by (2.8). Since

$$
d(f, F) \leq \frac{1}{1-L} d(f, J f) \leq \frac{L}{8(1-L)}
$$

the inequality (2.7) holds. From the definition of $F(x),(2.1)$ and $(2.6)$, we have

$$
\begin{aligned}
\| D F & (x, y, z, w) \| \\
= & \lim _{n \rightarrow \infty} \| 2^{n-1}\left(D f\left(\frac{x}{2^{n}}, \frac{y}{2^{n}}, \frac{z}{2^{n}}, \frac{w}{2^{n}}\right)-D f\left(-\frac{x}{2^{n}},-\frac{y}{2^{n}},-\frac{z}{2^{n}},-\frac{w}{2^{n}}\right)\right) \\
& +\frac{4^{n}}{2}\left(D f\left(\frac{x}{2^{n}}, \frac{y}{2^{n}}, \frac{z}{2^{n}}, \frac{w}{2^{n}}\right)+D f\left(-\frac{x}{2^{n}},-\frac{y}{2^{n}},-\frac{z}{2^{n}},-\frac{w}{2^{n}}\right)\right) \| \\
\leq & \lim _{n \rightarrow \infty} \frac{2^{n}+4^{n}}{2}\left(\varphi\left(\frac{x}{2^{n}}, \frac{y}{2^{n}}, \frac{z}{2^{n}}, \frac{w}{2^{n}}\right)+\varphi\left(-\frac{x}{2^{n}},-\frac{y}{2^{n}},-\frac{z}{2^{n}},-\frac{w}{2^{n}}\right)\right) \\
\leq & \lim _{n \rightarrow \infty} \frac{\left(2^{n}+4^{n}\right) L^{n}}{2 \cdot 4^{n}}(\varphi(x, y, z, w)+\varphi(-x,-y,-z,-w)) \\
= & 0
\end{aligned}
$$

for all $x, y, z, w \in V \backslash\{0\}$. By Lemma 2.2, $F$ is quadratic-additive.

Remark 2.6. If $\varphi$ satisfies the additional condition $\varphi(x, y, z, w)=\varphi(-x,-y,-z,-w)$ for all $x, y, z, w \in V \backslash\{0\}$ in Theorem 2.5, then we get

$$
\|f(x)-J f(x)\| \leq \frac{L}{16}(\varphi(x, x, x,-x)+\varphi(-x,-x,-x, x))
$$

for all $x \in V \backslash\{0\}$. It means that $d(f, J f) \leq \frac{L}{16}<\infty$ by the definition of $d$. Therefore the inequality (2.7) can be replaced by the inequality

$$
\|f(x)-F(x)\| \leq \frac{L \varphi(x, x, x,-x)}{8(1-L)}
$$

for all $x \in V \backslash\{0\}$.

\section{Applications}

For $f: V \rightarrow Y$, let us define

$$
\begin{aligned}
& A f(x, y):=f(x+y)-f(x)-f(y) \\
& Q f(x, y):=f(x+y)+f(x-y)-2 f(x)-2 f(y)
\end{aligned}
$$

for all $x, y \in V$. Using Theorem 2.3 and Theorem 2.5, we will show the stability results of the additive functional equation $A f \equiv 0$ and the quadratic functional equation $Q f \equiv 0$.

Corollary 3.1. Let $f_{i}: V \rightarrow Y, i=1,2$, be given for which there exist functions $\phi_{i}: V^{2} \rightarrow[0, \infty), i=1,2$, such that 


$$
\left\|A f_{i}(x, y)\right\| \leq \phi_{i}(x, y)
$$

for all $x, y \in V$, respectively. If there exists $L<1$ such that

$$
\begin{aligned}
& \phi_{1}(2 x, 2 y) \leq 2 L \phi_{1}(x, y), \\
& L \phi_{2}(2 x, 2 y) \geq 4 \phi_{2}(x, y)
\end{aligned}
$$

for all $x, y \in V$, then we have unique additive mappings $F_{i}: V \rightarrow Y, i=1,2$, such that

$$
\begin{aligned}
\left\|f_{1}(x)-f_{1}(0)-F_{1}(x)\right\| & \leq \frac{3\left(\Phi_{1}(x)+2\left\|f_{1}(0)\right\|\right)}{16\left(1-\max \left\{L, \frac{1}{2}\right\}\right)}, \\
\left\|f_{2}(x)-F_{2}(x)\right\| & \leq \frac{L \Phi_{2}(x)}{8(1-L)}
\end{aligned}
$$

for all $x \in V$, where $\Phi_{i}: V \rightarrow Y, i=1,2$, are defined by

$$
\begin{aligned}
\Phi_{i}(x):= & \phi_{i}(2 x, 0)+\phi_{i}(-2 x, 0)+2 \phi_{i}(x, x) \\
& +2 \phi_{i}(x,-x)+2 \phi_{i}(-x, x)+2 \phi_{i}(-x,-x)
\end{aligned}
$$

for all $x \in V$. In particular, the mappings $F_{1}, F_{2}$ are represented by

$$
\begin{aligned}
& F_{1}(x)=\lim _{n \rightarrow \infty} \frac{f_{1}\left(2^{n} x\right)}{2^{n}}, \\
& F_{2}(x)=\lim _{n \rightarrow \infty} 2^{n} f_{2}\left(\frac{x}{2^{n}}\right)
\end{aligned}
$$

for all $x \in V$. Moreover, if $0<L<\frac{1}{2}$ and $\phi_{1}(x, y)$ is continuous, then $f_{1}$ is itself an additive mapping.

Proof. Notice that

$$
D f_{i}(x, y, z, w)=A f_{i}(x+y, z+w)-A f_{i}(x, z)-A f_{i}(x, w)-A f_{i}(y, z)-A f_{i}(y, w)
$$

for all $x, y, z, w \in V$ and $i=1,2$. Put

$$
\varphi_{i}(x, y, z, w):=\phi_{i}(x+y, z+w)+\phi_{i}(x, z)+\phi_{i}(x, w)+\phi_{i}(y, z)+\phi_{i}(y, w)
$$

for all $x, y, z, w \in V$ and $i=1,2$, then $\varphi_{1}$ satisfies (2.2) and $\varphi_{2}$ holds (2.6). Therefore, according to Theorem 2.3, there exists a unique mapping $F_{1}: V \rightarrow Y$ satisfying (3.4), which is represented by (2.5). Observe that, by (3.1) and (3.2),

$$
\begin{aligned}
\lim _{n \rightarrow \infty}\left\|\frac{f_{1}\left(2^{n} x\right)+f_{1}\left(-2^{n} x\right)}{2^{n+1}}\right\| & =\lim _{n \rightarrow \infty}\left\|\frac{f_{1}\left(2^{n} x\right)+f_{1}\left(-2^{n} x\right)-f_{1}(0)}{2^{n+1}}\right\| \\
& =\lim _{n \rightarrow \infty} \frac{1}{2^{n+1}}\left\|A f_{1}\left(2^{n} x,-2^{n} x\right)\right\|
\end{aligned}
$$




$$
\begin{aligned}
& \leq \lim _{n \rightarrow \infty} \frac{1}{2^{n+1}} \phi_{1}\left(2^{n} x,-2^{n} x\right) \\
& \leq \lim _{n \rightarrow \infty} \frac{L^{n}}{2} \phi_{1}(x,-x)=0
\end{aligned}
$$

as well as

$$
\lim _{n \rightarrow \infty}\left\|\frac{f_{1}\left(2^{n} x\right)+f_{1}\left(-2^{n} x\right)}{2 \cdot 4^{n}}\right\| \leq \lim _{n \rightarrow \infty} \frac{2^{n} L^{n}}{2 \cdot 4^{n}} \phi_{1}(x,-x)=0
$$

for all $x \in V$. From this and (2.5), we get (3.6). Moreover, we have

$$
\left\|\frac{A f_{1}\left(2^{n} x, 2^{n} y\right)}{2^{n}}\right\| \leq \frac{\phi_{1}\left(2^{n} x, 2^{n} y\right)}{2^{n}} \leq L^{n} \phi_{1}(x, y)
$$

for all $x, y \in V$. Taking the limit as $n \rightarrow \infty$ in the above inequality, we get

$$
A F_{1}(x, y)=0
$$

for all $x, y \in V$. In particular, consider the case $0<L<\frac{1}{2}$ such that $\phi_{1}(x, y)$ is continuous, then $\varphi_{1}(x, y, z, w)$ is continuous on $(V \backslash\{0\})^{4}$ and we can say that $f_{1} \equiv F_{1}$ by Theorem 2.3 .

On the other hand, according to Theorem 2.5, there exists a unique mapping $F_{2}: V \rightarrow Y$ satisfying (3.5) which is represented by (2.8). Observe that

$$
\begin{aligned}
\lim _{n \rightarrow \infty} 2^{2 n-1}\left\|f_{2}\left(\frac{x}{2^{n}}\right)+f_{2}\left(\frac{-x}{2^{n}}\right)\right\| & =\lim _{n \rightarrow \infty} 2^{2 n-1}\left\|A f_{2}\left(\frac{x}{2^{n}},-\frac{x}{2^{n}}\right)\right\| \\
& \leq \lim _{n \rightarrow \infty} 2^{2 n-1} \phi_{2}\left(\frac{x}{2^{n}},-\frac{x}{2^{n}}\right) \\
& \leq \lim _{n \rightarrow \infty} \frac{L^{n}}{2} \phi_{2}(x,-x)=0
\end{aligned}
$$

as well as

$$
\lim _{n \rightarrow \infty} 2^{n-1}\left\|f_{2}\left(\frac{x}{2^{n}}\right)+f_{2}\left(\frac{-x}{2^{n}}\right)\right\| \leq \lim _{n \rightarrow \infty} \frac{L^{n}}{2^{n+1}} \phi_{2}(x,-x)=0
$$

for all $x \in V$. From this and (2.8), we get (3.7). Moreover, we have

$$
\left\|2^{n} A f_{2}\left(\frac{x}{2^{n}}, \frac{y}{2^{n}}\right)\right\| \leq 2^{n} \phi_{2}\left(\frac{x}{2^{n}}, \frac{y}{2^{n}}\right) \leq \frac{L^{n}}{2^{n}} \phi_{2}(x, y)
$$

for all $x, y \in V$. Taking the limit as $n \rightarrow \infty$ in the above inequality, we get

$$
A F_{2}(x, y)=0
$$

for all $x, y \in V$.

Corollary 3.2. Let $\phi_{i}: V^{2} \rightarrow[0, \infty), i=1,2$, be given functions. Suppose that each $f_{i}: V \rightarrow Y, i=1,2$, satisfies

$$
\left\|Q f_{i}(x, y)\right\| \leq \phi_{i}(x, y)
$$


for all $x, y \in V$, respectively. If there exists $0<L<1$ such that the mapping $\phi_{1}$ has the property (3.2) and $\phi_{2}$ holds (3.3) for all $x, y \in V$, then we have unique quadratic mappings $F_{1}, F_{2}: V \rightarrow Y$ such that

$$
\begin{aligned}
& \left\|f_{1}(x)-F_{1}(x)\right\| \leq \frac{3\left(\Phi_{1}(x)+4\left\|f_{1}(0)\right\|\right)}{32\left(1-\max \left\{L, \frac{1}{2}\right\}\right)}, \\
& \left\|f_{2}(x)-F_{2}(x)\right\| \leq \frac{L \Phi_{2}(x)}{16(1-L)}
\end{aligned}
$$

for all $x \in V$, where $\Phi_{i}: V \rightarrow Y, i=1,2$, is defined by

$$
\begin{aligned}
\Phi_{i}(x):= & \phi_{i}(2 x, 0)+2 \phi_{i}(0,2 x)+2 \phi_{i}(x, x)+2 \phi_{i}(x,-x)+\phi_{i}(-2 x, 0) \\
& +2 \phi_{i}(0,-2 x)+2 \phi_{i}(-x,-x)+2 \phi_{i}(-x, x) .
\end{aligned}
$$

In particular, $F_{1}$ and $F_{2}$ are represented by

$$
\begin{aligned}
& F_{1}(x)=\lim _{n \rightarrow \infty} 4^{-n} f_{1}\left(2^{n} x\right), \\
& F_{2}(x)=\lim _{n \rightarrow \infty} 4^{n} f_{2}\left(2^{-n} x\right)
\end{aligned}
$$

for all $x \in V$. Moreover, if $0<L<\frac{1}{2}$ and $\phi_{1}(x, y)$ is continuous, then $f_{1}$ is itself a quadratic mapping.

Proof. Notice that

$$
\begin{aligned}
D f_{i}(x, y, z, w)= & \frac{1}{2}\left(Q f_{i}(x+y, z+w)+Q f_{i}(x+w, y+z)-Q f_{i}(x-z, y-w)\right) \\
& -Q_{i} f(x, z)-Q f_{i}(y, w)
\end{aligned}
$$

for all $x, y, z, w \in V$ and $i=1,2$. Put

$$
\begin{aligned}
\varphi_{i}(x, y, z, w):= & \frac{1}{2}\left(\phi_{i}(x+y, z+w)+\phi_{i}(x+w, y+z)+\phi_{i}(x-z, y-w)\right) \\
& +\phi_{i}(x, z)+\phi_{i}(y, w)
\end{aligned}
$$

for all $x, y, z, w \in V$ and $i=1,2$, then $\varphi_{1}$ satisfies (2.2) and $\varphi_{2}$ holds (2.6). So we have

$$
\left\|D f_{i}(x, y, z, w)\right\| \leq \varphi_{i}(x, y, z, w)
$$

for all $x, y, z, w \in V$ and $i=1,2$. According to Theorem 2.3, there exists a unique mapping $F_{1}: V \rightarrow Y$ satisfying (3.8) which is represented by (2.5). Observe that

$$
\begin{aligned}
\lim _{n \rightarrow \infty}\left\|\frac{f_{1}\left(2^{n} x\right)-f_{1}\left(-2^{n} x\right)}{2^{n+1}}\right\| & =\lim _{n \rightarrow \infty} \frac{1}{2^{n+1}}\left\|Q f_{1}\left(0,2^{n} x\right)\right\| \\
& \leq \lim _{n \rightarrow \infty} \frac{1}{2^{n+1}} \phi_{1}\left(0,2^{n} x\right)
\end{aligned}
$$




$$
\leq \lim _{n \rightarrow \infty} \frac{L^{n}}{2} \phi_{1}(0, x)=0
$$

as well as

$$
\lim _{n \rightarrow \infty}\left\|\frac{f_{1}\left(2^{n} x\right)-f_{1}\left(-2^{n} x\right)}{2 \cdot 4^{n}}\right\| \leq \lim _{n \rightarrow \infty} \frac{L^{n}}{2^{n+1}} \phi_{1}(0, x)=0
$$

for all $x \in V$. From this and (2.5), we get (3.10) for all $x \in V$. Moreover, we have

$$
\left\|\frac{Q f_{1}\left(2^{n} x, 2^{n} y\right)}{4^{n}}\right\| \leq \frac{\phi_{1}\left(2^{n} x, 2^{n} y\right)}{4^{n}} \leq \frac{L^{n}}{2^{n}} \phi_{1}(x, y)
$$

for all $x, y \in V$. Taking the limit as $n \rightarrow \infty$ in the above inequality, we get

$$
Q F_{1}(x, y)=0
$$

for all $x, y \in V$. In particular, consider the case $0<L<\frac{1}{2}$ such that $\phi_{1}(x, y)$ is continuous, then $\varphi_{1}(x, y, z, w)$ is continuous on $(V \backslash\{0\})^{4}$ and we can say that $f_{1} \equiv F_{1}$ by Theorem 2.3. On the other hand, according to Theorem 2.5, there exists a unique mapping $F_{2}: V \rightarrow Y$ satisfying (3.9) which is represented by (2.8). Observe that

$$
4^{n}\left\|-f_{2}\left(\frac{x}{2^{n}}\right)+f_{2}\left(-\frac{x}{2^{n}}\right)\right\|=4^{n}\left\|Q f_{2}\left(0, \frac{x}{2^{n}}\right)\right\| \leq 4^{n} \phi_{2}\left(0, \frac{x}{2^{n}}\right) \leq L^{n} \phi_{2}(0, x)
$$

for all $x \in V$. It leads us to get

$$
\lim _{n \rightarrow \infty} 4^{n}\left(f_{2}\left(\frac{x}{2^{n}}\right)-f_{2}\left(-\frac{x}{2^{n}}\right)\right)=0 \text { and } \lim _{n \rightarrow \infty} 2^{n}\left(f_{2}\left(\frac{x}{2^{n}}\right)-f_{2}\left(-\frac{x}{2^{n}}\right)\right)=0
$$

for all $x, y \in V$. From these and (2.8), we obtain (3.11). Moreover, we have

$$
\left\|4^{n} Q f_{2}\left(\frac{x}{2^{n}}, \frac{y}{2^{n}}\right)\right\| \leq 4^{n} \phi_{2}\left(\frac{x}{2^{n}}, \frac{y}{2^{n}}\right) \leq L^{n} \phi_{2}(x, y)
$$

for all $x, y \in V$. Taking the limit as $n \rightarrow \infty$ in the above inequality, we get

$$
Q F_{2}(x, y)=0
$$

for all $x, y \in V$.

Now, we obtain Hyers-Ulam-Rassias stability results in the framework of normed spaces using Theorem 2.3, Theorem 2.5, Remark 2.4, and Remark 2.6.

Corollary 3.3. Let $X$ be a normed space. Suppose that the mapping $f: X \rightarrow Y$ satisfies the inequality

$$
\|D f(x, y, z, w)\| \leq \theta\left(\|x\|^{p}+\|y\|^{p}+\|z\|^{p}+\|w\|^{p}\right)
$$

for all $x, y, z, w \in X \backslash\{0\}$, where $\theta \geq 0$ and $p \in(-\infty, 0) \cup(0,1) \cup(2, \infty)$. Then there exists a unique quadratic-additive mapping $F: X \rightarrow Y$ such that 


$$
\|f(x)-F(x)\| \leq \begin{cases}\frac{2 \theta}{2^{p}-4}\|x\|^{p} & \text { if } p>2, \\ \frac{2 \theta}{2-2^{p}}\|x\|^{p} & \text { if } 0<p<1\end{cases}
$$

for all $x \in X \backslash\{0\}$. Moreover if $p<0$, then $f$ is itself a quadratic-additive mapping.

Proof. It follows from Theorem 2.3, Theorem 2.5, Remark 2.4, and Remark 2.6, by putting

$$
\varphi(x, y, z, w):=\theta\left(\|x\|^{p}+\|y\|^{p}+\|z\|^{p}+\|w\|^{p}\right)
$$

for all $x, y, z, w \in X \backslash\{0\}$ with $L=2^{p-1}<1$ if $p<1, L=2^{2-p}<1$ if $p>2$, and $L^{\prime}=2^{-p}<1$ if $p>0$.

Corollary 3.4. Let $X$ be a normd space. Suppose that the mapping $f: X \rightarrow Y$ satisfies the inequality

$$
\|D f(x, y, z, w)\| \leq \theta\|x\|^{p}\|y\|^{q}\|z\|^{r}\|w\|^{s}
$$

for all $x, y, z, w \in X \backslash\{0\}$, where $\theta \geq 0$ and $p+q+r+s \in(-\infty, 0) \cup(0,1) \cup(2, \infty)$. Then there exists a unique quadratic-additive mapping $F: X \rightarrow Y$ such that

$$
\|f(x)-F(x)\| \leq \begin{cases}\frac{\theta\|x\|^{p+q+r+s}}{\left.22^{p+q+r+s}-4\right)} & \text { if } p+q+r+s>2, \\ \frac{\theta\|x\|^{p+q+r+s}}{2\left(2-2^{p+q+r+s}\right)} & \text { if } 0<p+q+r+s<1\end{cases}
$$

for all $x \in X \backslash\{0\}$. Moreover if $p+q+r+s<0$, then $f$ is itself a quadratic-additive mapping.

Proof. It follows from Theorem 2.3, Theorem 2.5, Remark 2.4, and Remark 2.6, by putting

$$
\varphi(x, y, z, w):=\theta\|x\|^{p}\|y\|^{q}\|z\|^{r}\|w\|^{s}
$$

for all $x, y, z, w \in X \backslash\{0\}$ with $L=2^{p+q+r+s-1}<1$ if $p+q+r+s<1, L=$ $2^{2-p-q-r-s}<1$ if $p+q+r+s>2$, and $L^{\prime}=2^{-p-q-r-s}<1$ if $p+q+r+s>0$.

\section{REFERENCES}

1. T. Aoki: On the stability of the linear transformation in Banach spaces. J. Math. Soc. Japan 2 (1950), 64-66.

2. L. Cădariu \& V. Radu: Fixed points and the stability of Jensen's functional equation. J. Inequal. Pure Appl. Math. 4 (2003), no. 1, Art. 4.

3. $\quad$ Fixed points and the stability of quadratic functional equations. An. Univ. Timisoara Ser. Mat.-Inform. 41 (2003), 25-48.

4. __ : On the stability of the Cauchy functional equation: a fixed point approach in Iteration Theory. Grazer Mathematische Berichte, Karl-Franzens-Universitäet, Graz, Graz, Austria 346 (2004), 43-52. 
5. I.-S. Chang, E.-H. Lee \& H.-M. Kim: On Hyers-Ulam-Rassias stability of a quadratic functional equation. Math. Inequal. Appl. 6 (2003), 87-95.

6. P. Găvruta: A generalization of the Hyers-Ulam-Rassias stability of approximately additive mappings. J. Math. Anal. Appl. 184 (1994), 431-436.

7. D. H. Hyers: On the stability of the linear functional equation. Proc. Natl. Acad. Sci. 27 (1941), 222-224.

8. G.-H. Kim: On the stability of functional equations with square-symmetric operation. Math. Inequal. Appl. 4 (2001), 257-266.

9. H.-M. Kim: On the stability problem for a mixed type of quartic and quadratic functional equation. J. Math. Anal. Appl. 324 (2006), 358-372.

10. Y.-H. Lee: On the stability of the monomial functional equation. Bull. Korean Math. Soc. 45 (2008), 397-403.

11. Y.-H. Lee \& K.-W. Jun: A generalization of the Hyers-Ulam-Rassias stability of Jensen's equation. J. Math. Anal. Appl. 238 (1999), 305-315.

12. _ _ A generalization of the Hyers-Ulam-Rassias stability of Pexider equation. $J$. Math. Anal. Appl. 246 (2000), 627-638.

13. __ A note on the Hyers-Ulam-Rassias stability of Pexider equation. J. Korean Math. Soc. 37 (2000), 111-124.

14. _ : On the stability of approximately additive mappings. Proc. Amer. Math. Soc. 128 (2000), 1361-1369.

15. B. Margolis \& J.B. Diaz: A fixed point theorem of the alternative for contractions on a generalized complete metric space. Bull. Amer. Math. Soc. 74 (1968), 305-309.

16. Th. M. Rassias: On the stability of the linear mapping in Banach spaces. Proc. Amer. Math. Soc. 72 (1978), 297-300.

17. I.A. Rus: Principles and applications of fixed point theory. Ed. Dacia, Cluj-Napoca. 1979(in Romanian).

18. S.M. Ulam: A collection of mathematical problems. Interscience, New York, 1968, p. 63.

a Department of Mathematics Education, Gonguu National University of Education, GonguU 314-711, KoreA

Email address: ssjin@gjue.ac.kr

${ }^{\text {b} D e p a r t m e n t ~ o f ~ M a t h e m a t i c s ~ E d u c a t i o n, ~ G o n g u u ~ N a t i o n a l ~ U n i v e r s i t y ~ o f ~ E d u c a t i o n, ~}$ GonguU 314-711, KoreA

Email address: yanghi2@hanmail.net 